\title{
Study on the Influence of Piloti Ratio on Residential Block's Wind Environment by Coupled Simulation Method
}

\author{
Tianyu $\mathrm{Xi}^{1}$, Jianhua Ding ${ }^{2}$ and Hong Jini ${ }^{1}$ \\ ${ }^{1}$ Architecture School of Harbin Institute of Technology, Harbin City, 150006, China \\ ${ }^{2}$ School of Architecture, Tsinghua University, Peking City, 100084, China
}

\begin{abstract}
The environmental degradation results in not only the increase of energy consumption in cities but also healthy problem of people, and many researches regarding to outdoor environment and thermal comfort are carried out. Wind velocity is considered to be one of the thermal comfort influential factors, and there are few work studied the comprehensive influence of piloti on wind environment by coupled simulation method, which offers high precision CFD simulation results. This paper taking the city of Guangzhou, China, as an example, adopting coupled simulation method, studied the wind environment of residential blocks under various piloti ratio $(0 \%, 40 \%, 60 \%, 80$ and $100 \%$ ). Both of the cumulative distribution and average wind velocity under piloti and out of piloti area are simulated and analysed. This work is expected to offer reference for architects and civil engineers in outdoor environment and thermal comfort design, and contribute to further study in sustainable and healthy building environment research field.
\end{abstract}

\section{Introduction}

The degradation of outdoor environment leads to not only energy consumption but also people's health problems. of Urban heat island is now regarded as one of the most serious environmental problems, and to optimize the outdoor thermal environment and thermal comfort, a lot of research were carried out [1]-[4], including plant, high reflective capability materials of building envelope, green building walls and roofs, improvement of city ventilation, use of sea wind and decrease of artificial heat, etc.

To get good outdoor ventilation and create shadow areas, piloti is commonly used in tropical and subtropical climate zones (Fig. 1), but there are few studies reveal the comprehensive influence of piloti on wind environment in residential blocks, and furthermore, a coupled simulation method, which offers high precision prediction result, is seldom taken into consideration in piloti research field. $\mathrm{Xi}$ et al. published field measurement results of various human built elements in Guangzhou [5]-[8], including piloti, but no systematic analysis by simulation was used in those research. The influence of piloti on mean radiant temperature simulated by $3-\mathrm{D}$ unsteady state heat balance radiation calculation method was published by $\mathrm{Xi}$, Hong Jin et al. but no wind environment was mentioned [9], [10]. Xi, Qiong Li et al. studied on the effects of semi-open space (100 percent ratio piloti) on the outdoor thermal environment of residential communities, by adopting coupled simulation method, but no different piloti ratio cases were compared [11].
This research takes residential blocks in Guangzhou, China as an example, aiming at study on the comprehensive influence of piloti ratio on the wind environment. A coupled simulation method, which combines the commercial STAR-cd software and homemade program (coded in Tokyo University) was adopted in this study, and this method is expected to get high precision prediction result.

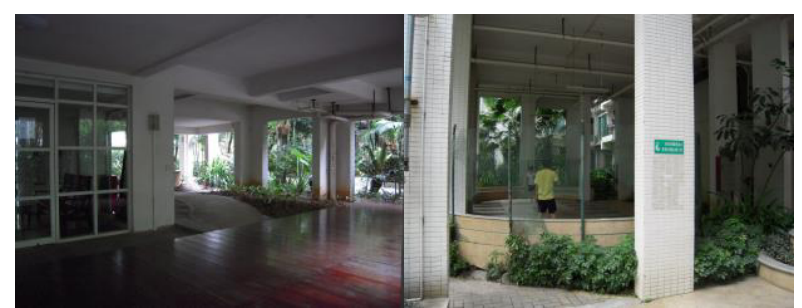

Figure 1. Building piloti design in subtropical climate zones.

\section{Methodology}

\subsection{Coupled simulation method}

Firstly, non-isothermal CFD analysis is carried out by STAR-cd, using data from local meteorological bureau (wind velocity and prevailing wind direction, and air temperature) and observed data (ground and building surface temperature). In this step, the surface temperature is set to be mean value as traditional method, which cannot reflect the real surface situation and will cause buoyance calculation omit in the velocity simulation. 
Secondly, ground and building surface temperatures are recalculated in step 2 by homemade program (coded in Tokyo University), which is based on unsteady state heat balance calculation including 3-dimentional radiation and 1-dimentional conduction calculations.

Based on outcomes in above 2 steps, CFD analysis is carried out one more time in STAR-cd and more accurate results will be obtained (Fig. 2).

\subsection{Time selected in simulation}

Based on TMY (typical meteorological year) data, 24hour meteorological data are averaged per day to get mean daily data. Consequently, the whole summer mean data are obtained by averaging the cumulated mean daily data. Finally, analysis date is determined by choosing the day whose data are most close to the whole summer mean data. Therefore, 14th July in Guangzhou is selected as a typical day of summer. Analysis time is selected based on at the time when maximum temperature occurred during analysis date (15:00)

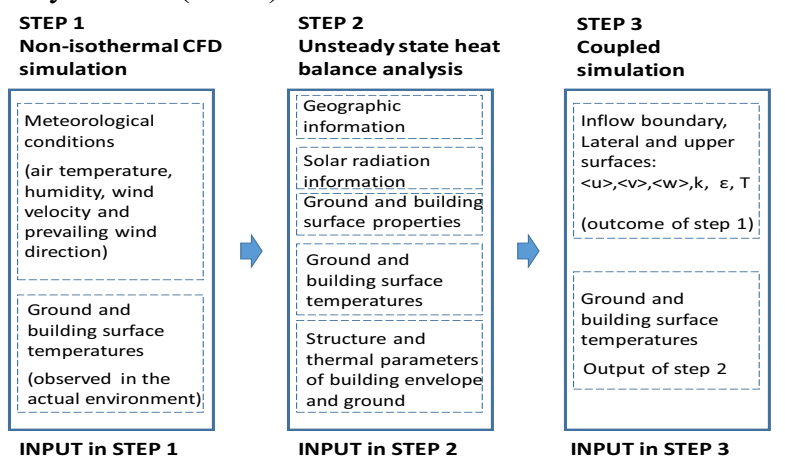

Figure 2. Coupled simulation chart flow.

\subsection{Cases design}

The domain size, wind direction and building orientation are shown in Fig. 3, the distance between buildings and inflow and wall boundary is set to be 5 times of building height, and the distance between buildings and outlet boundary is set to be 15 times of building height, both of which are set due to AIJ guidelines. The evaluated area is set in the middle of the community to avoid the lateral influence of CFD simulation. The piloti ratio of each case is set to be $0,40,60,80$ and 100 percent. To avoid the lateral influence, the standard area was chosen in the centre of simulated cases (Fig. 4).

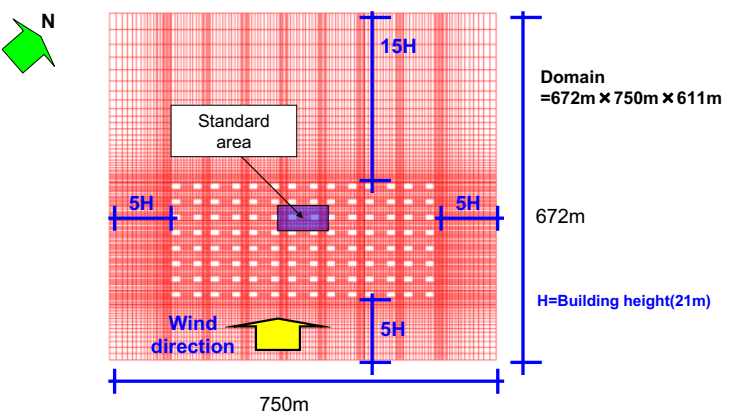

Figure 3. Domain description of simulation.

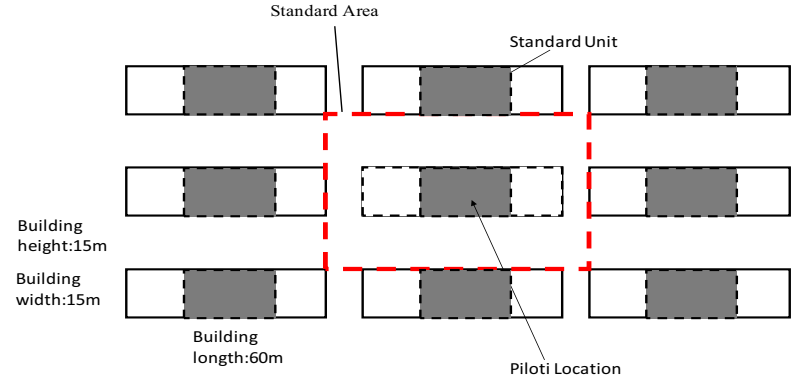

Figure 4. Standard unit and standard area.

\section{Results and analysis}

\subsection{Wind environment of non-piloti area}

The cumulative distribution of wind velocity of those non-piloti areas is shown in Fig. 6. It shows that when piloti ratio is lower than 40 percent, the average wind velocity is clearly divided into 2 levels, and the blank interval is vanished when piloti ratio reaches 60 percent, which should be because the non-piloti area includes wind channel area and space before and after buildings. When piloti ratio is 100 percent, no low wind velocity exists anymore, and the lowest wind velocity is over $0.9 \mathrm{~m} / \mathrm{s}$.

The average wind velocity of non-piloti area is plotted in Fig. 5, and it shows that 40 percent piloti ratio cannot optimize the wind environment efficiently, and the slop becomes shaper when piloti ratio increases from 60 to 80 percent, which seemed to be the inflection point of nonpiloti space wind velocity.

\subsection{Wind environment of piloti area}

The cumulative distribution of wind velocity of piloti areas is shown in Fig. 7, and the average wind velocity of non-piloti area is plotted in Fig. 8. When piloti ratio equals to $40 \%$, the wind velocity is very low, and no velocity is beyond $0.3 \mathrm{~m} / \mathrm{s}$. Each 20 percent piloti ratio increase can highly improve the wind velocity, and both of the lowest and highest wind velocity value keeps increasing along with the piloti ratio's change from $40 \%$ to $100 \%$. 80 percent and 100 percent piloti ratio can highly optimize the wind environment under piloti area, leaving blank in low velocity intervals (Fig.7). The increase slop changed at the $80 \%$ piloti ratio, and before that the increasing trend seemed to be constant.

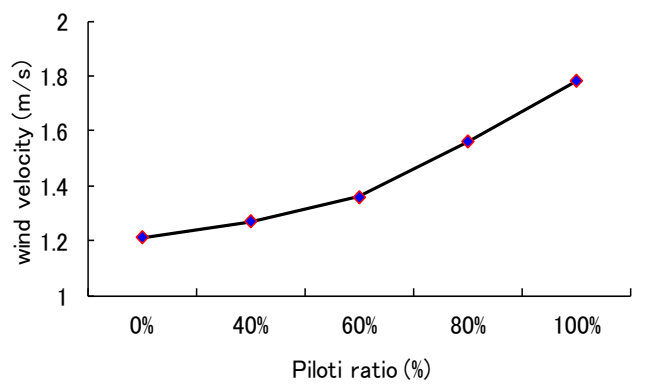

Figure 5. Average wind velocity of non-piloti area. 


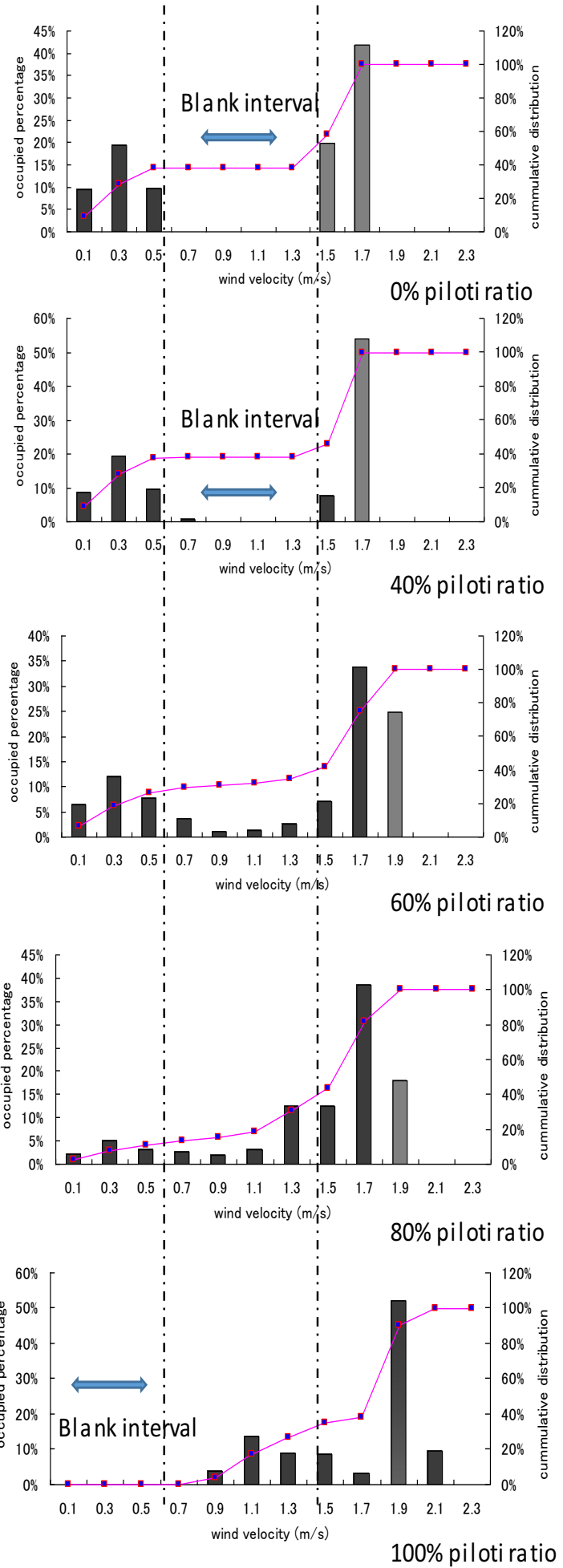

Figure 6. Wind velocity cumulative distribution of non-piloti area.

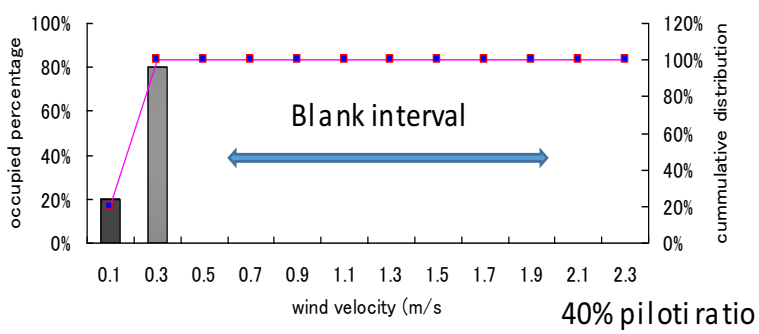

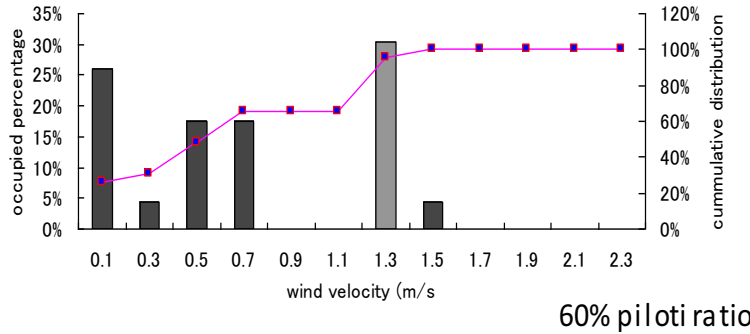
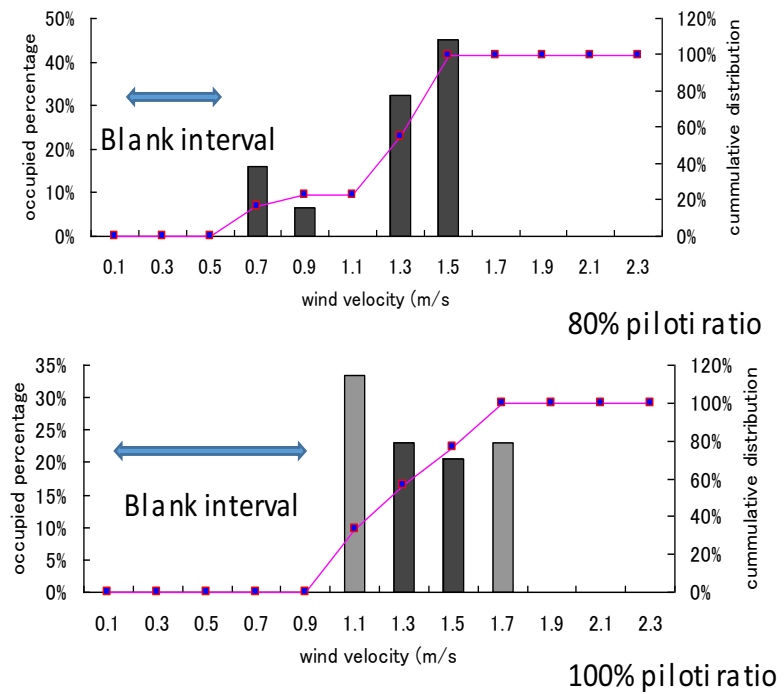

Figure 7. Wind velocity cumulative distribution of piloti area.

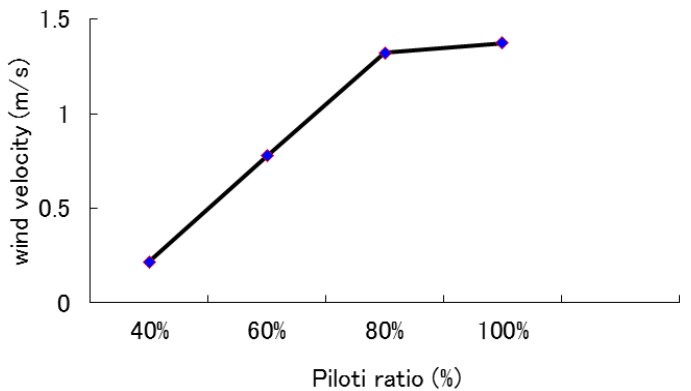

Figure 8. Average wind velocity of piloti area.

\section{Conclusions}

This work studied the influence of piloti ratio on residential block's wind environment by coupled simulation method, and to avoid the lateral influence, the standard area was chosen in the centre of simulated cases, which was divided into 2 parts to be analysed, the area under piloti and the space out of piloti.

For the non-piloti space, high wind velocity area (wind channel between buildings) and low wind area (before and after the buildings) are clear to be observed when piloti ratio is lower than 80 percent, and When piloti ratio is 100 percent, no low wind velocity exists anymore, and the lowest wind velocity is over $0.9 \mathrm{~m} / \mathrm{s}$. For the average wind velocity, the slop becomes shaper when piloti ratio increases from 60 to 80 percent, which seemed to be the inflection point of non-piloti space wind velocity.

For area under piloti, the optimization on wind environment of 40 percent piloti ratio is very limited, and 80 and 100 percent piloti ratio is recommended in 
subtropical climate cities, whose lowest wind velocity is $0.7 \mathrm{~m} / \mathrm{s}$ and $1.1 \mathrm{~m} / \mathrm{s}$ independently. The increase of velocity is constant before 80 percent, and only minus increase was observed when piloti ratio changed from 80 to 100 percent.

\section{Acknowledgement}

This work was financially supported by the National Natural Science Foundation of China (Grant No. 51408160), China Postdoctoral Science Foundation (2015M571419), and the Project-sponsored by SRF for ROCS, SEM. Work partially supported by the Fundamental Research Fund for the Central Universities (HIT.NSRIF.2014 075), Harbin Science and Technology Bureau (2015RQQXJ068) and Heilongjiang Province Postdoctoral Science Foundation(LBH-Z15084).

\section{References}

1. Tetsu Kubota, Supian Ahmad, Journal of Asian Architecture and Building Engineering, 199-206, (2006)

2. Uehara Kiyoshi, Ymamao Yukio, Oikawa Susumu and Mochida Akashi, Japan Society for Atmospheric Environment, 42 (5), (2007)

3. Takanobu Moriizumi, Toru Kawai, Atsushi Inagaki, Manabu Kanda, Paper Collection of Hydro Science and Hydraulic Engineering, 52, (2008)
4. Yoshida Shinji, Murakami Shuzo, Mochida Akashi et al. Paper Collection of Planning System of Annual Meeting of Architectural Institute of Japan, No. 529, 77-84, (2003)

5. Tianyu Xi, Qiong Li, Akashi Mochida, Qinglin Meng, Journal of Habitat Engineering, 3(2), 253 260, (2011)

6. Tianyu Xi, Qiong Li, Akashi Mochida, Qinglin Meng, Building and Environment, 52, 162-170, (2012)

7. Tianyu Xi, Qiong $\mathrm{Li}$ and Akashi Mochida, Summaries of Technical Papers of Annual Meeting of Architectural Institute of Japan, Environmental Engineering, 805-806, (2011)

8. Tianyu Xi, Qiong Li, Akashi Mochida and Qinglin Meng, 4th International Conference on HumanEnvironment System, ICHES2011 in Sapporo, Japan, 85-90, (2011)

9. Tianyu Xi, Jianhua Ding and Hongjin, Journal of Harbin Institute of Technology, 20 (4), 91-95 (2014)

10. Tianyu $\mathrm{Xi}$, Hong Jin and Jianhua Ding, International Conference on Materials Engineering and Environment Science, MEES2015 in Wuhan, China, 478-485, (2015)

11. Tianyu Xi, Yingli Xuan and Akashi Mochida, Technical papers of Annual Meeting of Society of Heating, Air-conditioning and Sanitary Engineers of Japan, 405-408, (2009) 